\title{
An algorithm to measure unsymmetrical circle shape of intravascular ultrasound image using image processing techniques
}

\author{
Suhaili Beeran Kutty ${ }^{1}$, Rahmita Wirza O.K Rahmat ${ }^{2}$, Sazzli Shahlan Kassim ${ }^{3}$, Hizmawati Madzin ${ }^{4}$, \\ Hazlina Hamdan ${ }^{5}$ \\ 1,2,4,5 Faculty of Computer Science and Information Technology, Universiti Putra Malaysia, Serdang, Malaysia \\ ${ }^{3}$ Faculty of Medicine, Universiti Teknologi MARA, Sungai Buloh, Malaysia \\ ${ }^{1}$ Faculty of Electrical Engineering, Universiti Teknologi MARA, Shah Alam, Malaysia
}

\begin{abstract}
Article Info
Article history:

Received Nov 5, 2019

Revised Jan 12, 2020

Accepted May 19, 2020

\section{Keywords:}

Border detection

IVUS measurement

Maximum diameter

Minimum diameter

ABSTRACT

In diagnosing coronary artery disease, measurement of the cross-sectional area of the lumen, maximum and minimum diameter is very important. Mainly, it will be used to confirm the diagnosing, to predict the stenosis if any and to ensure the size of the stent to be used. However, the measurement only offers by the existing software and some of the software needs human interaction to complete the process. The purpose of this paper is to present the algorithm to measure the region of interest (ROI) on intravascular ultrasound (IVUS) using an image processing technique. The methodology starts with image acquisition process followed by image segmentation. After that, border detection for each ROI was detected and the algorithm was applied to calculate the corresponding region. The result shows that the measurement is accurate and could be used not only for IVUS but applicable to solid circle and unsymmetrical circle shape.
\end{abstract}

Unsymmetrical circle
This is an open access article under the CC BY-SA license.

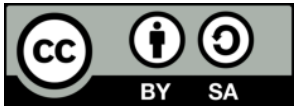

\section{Corresponding Author:}

Suhaili Beeran Kutty, Faculty of Electrical Engineering, Universiti Teknologi MARA, 40450 Shah Alam, Selangor, Malaysia.

Email: suhaili88@uitm.edu.my

\section{INTRODUCTION}

Heart disease refers to many heart-related problems and conditions. In early diagnosing, electrocardiograph (ECG) is used to to identify, examine and provide valuable data of the heart [1]. The distribution of mortality rates caused by heart disease widespread all over the world. In Malaysia, ischaemic heart diseases were the principal cause of death in 2016 [2]. Ischaemic heart diseases are also known as Coronary Artery Disease (CAD) or coronary heart disease and it caused 1 of every 7 deaths in the United States in 2011 [3]. Recently, the American Heart Association (AHA) reported that the ratio of the mortality rate caused by the CAD in 2014 is similar to 2011 [4]. This is a universal issue because the number of CAD cases increases steadily in both genders, men, and women [5].

To diagnose CAD, coronary angiography is used to depict the presence of stenosis in the coronary artery. However, coronary angiography only shows the silhouette of the inner layer of the coronary artery [6, 7]. Then, intravascular ultrasound (IVUS) technology is the medical imaging modality used to show the inner structure of the artery [8,9]. Figure 1 shows the sample image of coronary angiography and IVUS. In the catheterization lab, both modalities were fused together mentally by clinical experts because the modalities 
were displayed separately either on a different screen or on the same screen but in different windows. To overcome this problem, medical image registration was used to combine both modalities [10-12]. The registration of the coronary artery and IVUS starts with the image acquisition process where the IVUS and coronary angiography of the same patient were collected. The coronary angiography image is used to construct the trajectory. In IVUS, the lumen area and media adventitia were segmented to form the inner wall of the artery. Then, corresponding points were identified, and image registration was performed. Next, threedimensional reconstruction steps were applied to build the model. Finally, the size of the artery including the area, the minimum and maximum diameter of the lumen were computed. The measurement of the coronary artery size provided by the fusion can ease the CAD diagnosis process especially in determining the stenosis area and the size of the balloon that could be used. More than that, [13] has outlined the guidelines for implementing IVUS interpretation via lumen cross-sectional area (CSA), maximum and minimum lumen diameter. Based on these three important measurements, other measurements could be defined such as the lumen eccentricity and atheroma cross-sectional area which is vital to help CAD diagnosis process [14].

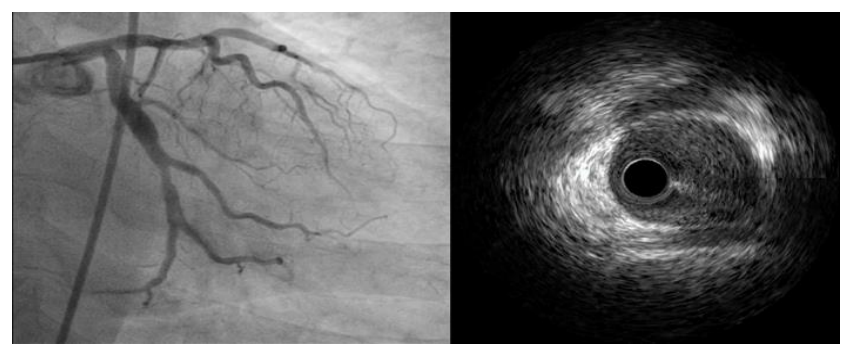

Figure 1. Coronary angiography and IVUS images taken with approval from Catheterization Lab, UiTM Sg. Buloh, Selangor, Malaysia

However, in many cases, the measurement of the area, minimum, and maximum of the area are not measured as mentioned in [15] accept the work done by [12]. It is supposedly, after the IVUS modality was segmented, the measurement of the IVUS should be recorded to provide valuable information for the specialist to diagnose the artery. This is because the gold standard modality, coronary angiography only displays the silhouette of the lumen and the specialist could interpret the percentage of the stenosis based on the image but it will lead to variability interpretation [16]. To assess the IVUS, the specialist obtained simple diameter measurement through the imaging package that they used and some application need human intervention $[17,18]$. A sample of the measurement provided by the imaging package is presented in Figure 2. So, in this study, we provide the measurement of the coronary artery cross-sectional image based on the image processing technique. The image processing technique is referred to the process that the image is utilized including pre-processing, detect a region of interest or features and analyzed the image [19]. The important of image processing technique to medical imaging is it can provide solution if the available software has limitation operations in diagnosing the image [20].

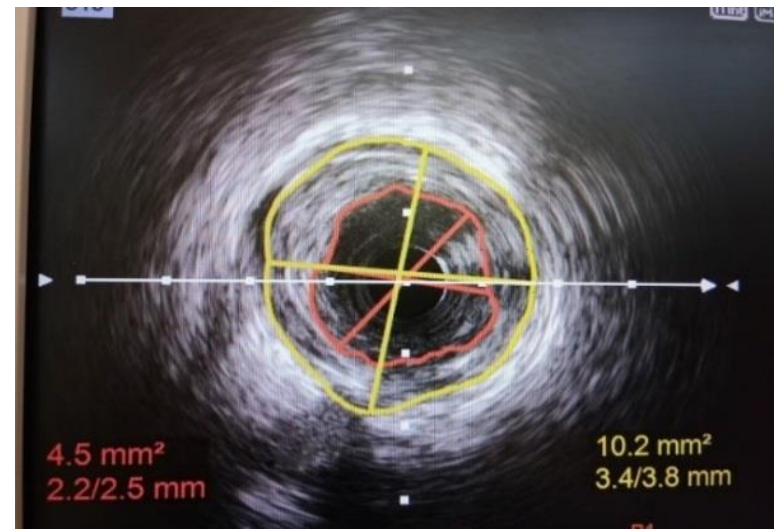

Figure 2. The measurement of CSA, minimum and maximum diameter provided by medical imaging software 


\section{RESEARCH METHOD}

\subsection{Lumen cross sectional area measurement}

IVUS provided us with excellent detailed images of the artery's interior walls. After important areas on the IVUS were detected during the segmentation process which was lumen and media-adventitia, it is significant to measure that area. According to [21], the calculation CSA of the lumen, minimum and maximum diameter were more accurate after computational segmentation process on IVUS was done. Furthermore, the information provided by the IVUS measurement serves to confirm the finding by others modalities such as x-ray angiography or CTA [17]. Lumen CSA is an important area to be measured in assessing the IVUS image. Besides that, this area also used to compare the precision of the segmentation approaches. The lumen shape is unsymmetrical circle. Figure 3 shows the sample of the lumen CSA.

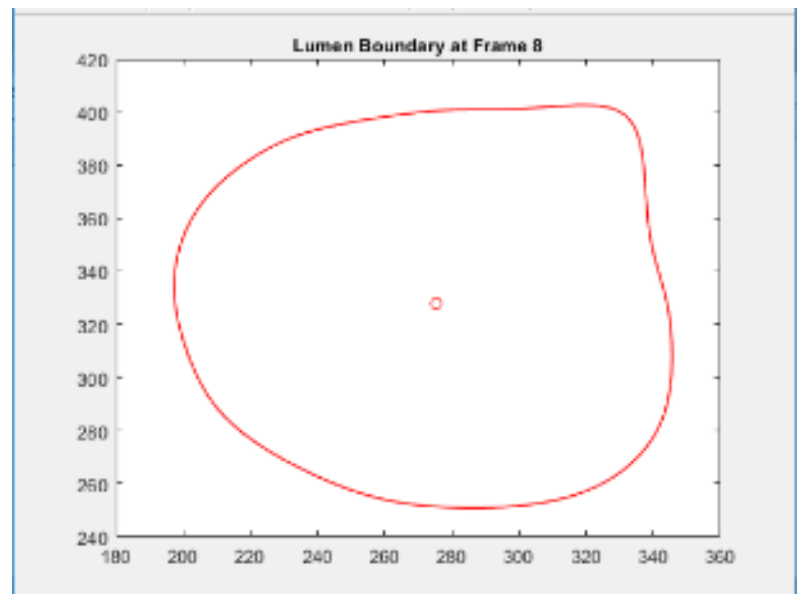

Figure 3. A sample shape of lumen CSA after the segmentation process

The steps to measure the CSA of the lumen for each slice of IVUS are as follow:

Step 1: Get the number of the points as $\mathrm{n}$ from the points ( $\mathrm{x}$ and $\mathrm{y}$ ) of the lumen border that we recorded after the segmentation process.

Step 2: Set one variable for the area, areaVar $=0$. We need to give 0 as an initial value to the areaVar to avoid the 'unrecognized' value to be added to the summation.

Step 3: After that, the formula as in (1) is used to calculate the CSA of the lumen. The measurement is in pixels.

$$
\text { areaVar }=\sum_{i=1}^{n} \frac{\left(x_{i} y_{i+1}-y_{i} x_{i+1}\right)}{2}
$$

\subsection{Computation of minimum and maximum diameter for unsymmetrical circle shape}

Because the shape of the lumen is not the solid circle, the measurement of minimum and maximum diameter of the shape is necessary. The minimum and maximum diameter of the lumen were used to confirm the existence of the stenosis and to predict the percentage.

The steps to compute the minimum and maximum diameter are as follow:

Step 1: Get $n$ points, $(x, y)$ of the lumen border that we stored after the segmentation process.

Step 2: Determine the centroid $\left(C_{x}, C_{y}\right)$ for the points $x$ and $y$ as in (2) and (3) respectively.

$$
\begin{aligned}
& C x=\sum_{i=1}^{n} \frac{x_{i}+x_{i+1}}{n} \\
& C y=\sum_{i=1}^{n} \frac{y_{i}+y_{i+1}}{n}
\end{aligned}
$$


where $\mathrm{x} \_1, \mathrm{x} \_2, \mathrm{x} \_3, \ldots \mathrm{x} \_\mathrm{n}$ and $\mathrm{y} \_1, \mathrm{y} \_2, \mathrm{y} \_3, \ldots \mathrm{y} \_\mathrm{n}$ denote a set of $\mathrm{n}$ points and $\mathrm{x} \_\mathrm{i}$ and $\mathrm{y} \_\mathrm{i}$ represents the ith number in the set of points.

Step 3: Get the radius, $r$ for each point using the formula in (4).

$$
r_{i}=\sqrt{\left(x_{i}-c_{x}\right)^{2}+\left(y_{i}-c_{y}\right)^{2}}
$$

Step 4: Determine the angle of each point using (5) for $x$ and (6) for $y$.

$$
\begin{aligned}
\cos \theta_{i} & =\frac{x_{i}-c_{x}}{r_{i}} \\
\sin \theta_{i} & =\frac{y_{i}-c_{y}}{r_{i}}
\end{aligned}
$$

Step 5: Sort the points from $0^{\circ}$ to $360^{\circ}$.

Step 6: Match the points where the angles are below than $180^{\circ}$ to the points that have angles more than $180^{\circ}$ with the order.

Step 7: Add the radius, $r$ of each matched point.

Step 8: Determine the minimum diameter, $\min D$ and maximum diameter, $\max D$.

Step 9: Specify the calibration factor (pixels $\rightarrow \mathrm{mm}$ )

Step 10: Change the measurement from pixels to millimeter $(\mathrm{mm})$ by multiply the pixels with the calibration factor.

The calibration factor is used to change the measurement from pixels to the millimeter. In this study, the calibration factor was determined using the IVUS image itself. Using the DICOM viewer software to view the IVUS image, we traced the diameter of the catheter. The diameter of the catheter was chosen because we already know the real measurement is $1.0 \mathrm{~mm}$. In pixels, the diameter is equal to 54 pixels. To get the calibration factor, $1.0 \mathrm{~mm}$ is divided by 54 pixels and the answer is $0.0176 \mathrm{~mm}$. To ensure our calibration factor is accurate, pixel spacing entity in the DICOM file was checked and the value is 0.01755671 which is similar to the value of the calibration factor. Figure 4 depicted the diameter of the catheter and the value of pixel spacing in the DICOM file.

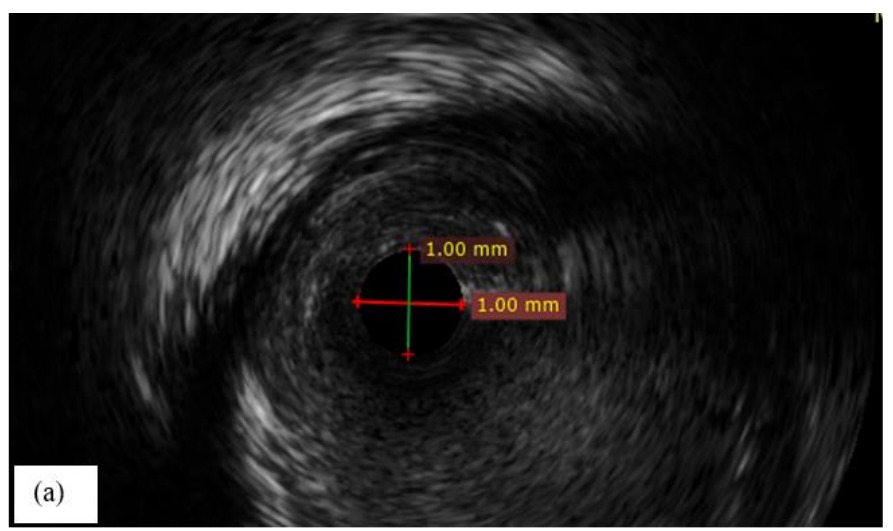

\begin{tabular}{|llllll|}
\hline 0028,0010) & US & 1 & 2 & Rows & 512 \\
0028,0011) & US & 1 & 2 & Columns & 512 \\
\hline 028,0030$)$ & DS & 2 & 22 & Pixel Spacing & 0.0175567110 .01755671 \\
D028,0100) & US & 1 & 2 & Bits Allocated & 8 \\
\hline (b) & US & 1 & 2 & Bits Stored & 8 \\
\hline
\end{tabular}

Figure 4. (a) The diameter of the catheter is $1.0 \mathrm{~mm}$, (b) The value of the pixel spacing in the DICOM file

Figure 5 shows the example of how the diameter is computed where the green indicates minimum diameter and blue is the maximum diameter. 


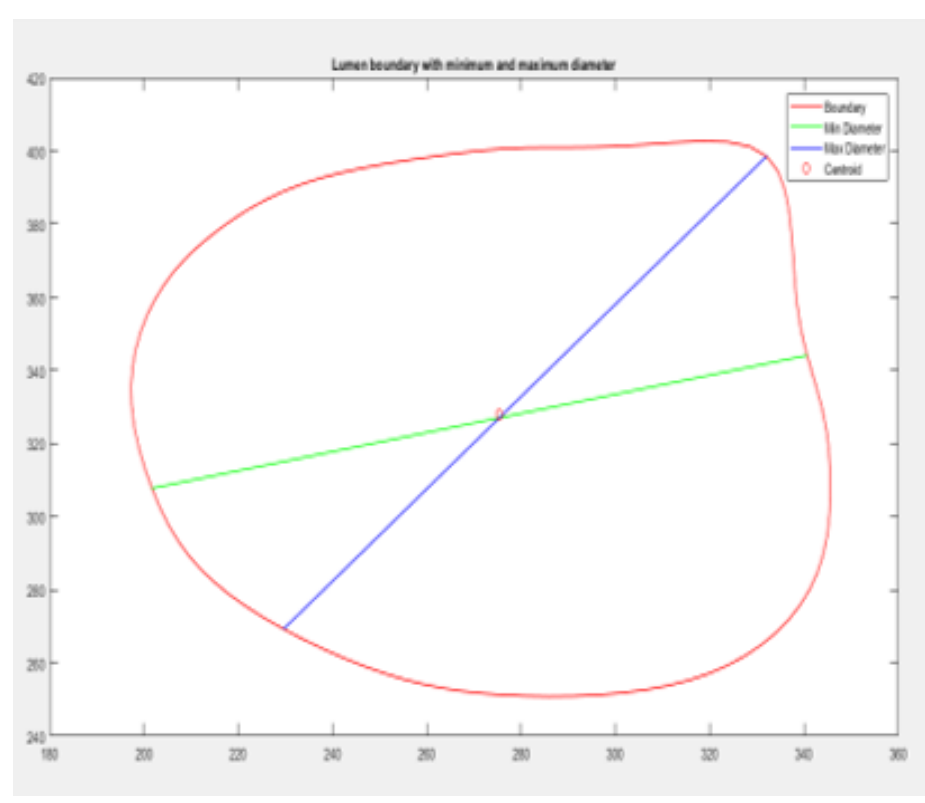

Figure 5. The computation of minimum and a maximum diameter of the lumen boundary

\section{RESULTS AND DISCUSSION}

\subsection{Experiment on the symmetry circle object}

The validation process is very important to verify that the algorithm is accurate. As example,to evaluate the experiment, [22] used to compare their findings with experts based on the accuracy scale. In this work, to ensure the computation is accurate, the algorithm was tested using coins that have a symmetrical circle shape and the diameter has been stated by Bank Negara Malaysia (BNM). The experiment started by specifying the value of the calibration factor. In this experiment, mobile handphone with camera 12 MP is used where the focal length is $3.7 \mathrm{~mm}$. The calibration factor is 69.4 pixels where the length for $5 \mathrm{~cm}$ is equal to 347 pixels. Four different types of coins are used in this experiment as shown in Table 1 .

Table 1. Type of coins with diameter properties specified by BNM

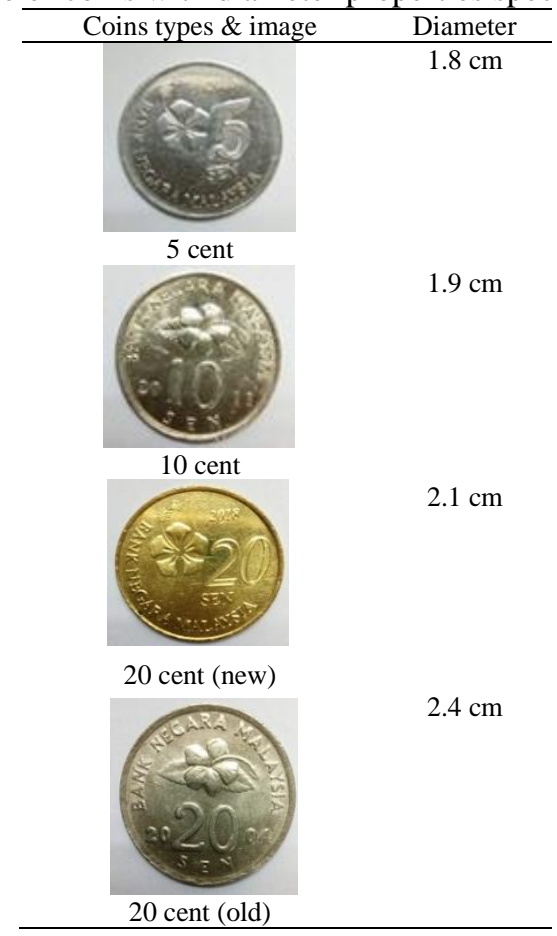


The coins have been segmented and the boundary of each coin was detected and recorded in $x$ and $y$ coordinate. The proposed algorithm to calculate the diameter was used. Table 2 shows the finding. For each type of the coins, three different coins were selected randomly and the diameter was recorded in pixels (px) before it was transferred to centimeter $(\mathrm{cm})$ using the calibration factor.

Table 2. Diameter measurement of the coins using the proposed method

\begin{tabular}{ccccccc}
\hline Coins & \multicolumn{2}{c}{$1 \mathrm{st}$} & \multicolumn{2}{c}{ 2nd } & \multicolumn{2}{c}{ 3rd } \\
\hline Units & $\mathrm{px}$ & $\mathrm{cm}$ & $\mathrm{px}$ & $\mathrm{cm}$ & $\mathrm{px}$ & $\mathrm{cm}$ \\
\hline 5 cent & 126.9285 & 1.8289 & 125.5650 & 1.8091 & 131.6065 & 1.8963 \\
10 cent & 133.4198 & 1.9225 & 142.3238 & 2.0508 & 130.7817 & 1.8845 \\
20 cent (new) & 140.5405 & 2.0251 & 147.1124 & 2.1198 & 146.8579 & 2.1161 \\
20 cent (old) & 168.6012 & 2.4294 & 169.6954 & 2.4452 & 173.9292 & 2.506 \\
\hline
\end{tabular}

A one-sample t-test is a method used to compare either the findings are acceptable or not. The null hypothesis and the alternative hypothesis used in this comparison stated as below:

Null Hypothesis, Ho:

Alternative Hypothesis, $\mathrm{Ha}$ :
There is no difference between the diameter computed by the proposed algorithm with the diameter specified by the BNM

There is a specific difference between the diameter computed by the proposed algorithm with the diameter specified by BNM

The result of the t-test as shown in Table 3. Therefore significant p-value is more than 0.05 for all types of coins used, hence it can be concluded that it accept the null hypothesis, Ho which implies that the diameter, measured by the proposed algorithm is almost similar to the real value specified by the BNM. In conclusion, our proposed algorithm to compute the diameter is accurate and can be used in practice.

Table 3. One sample t-test in comparing a data set of diameters for four types of coin to a diameter value specified by BNM

\begin{tabular}{|c|c|c|c|c|}
\hline & 5 cents & 10 cents & 20 cent (new) & 20 cent (old) \\
\hline Mean & 1.844766667 & 1.9526 & 2.087 & 2.4602 \\
\hline Variance & 0.002089773 & 0.00759343 & 0.00287713 & 0.00163564 \\
\hline Observations & 3 & 3 & 3 & 3 \\
\hline Hypothesized Mean & 1.8 & 1.9 & 2.1 & 2.4 \\
\hline df & 2 & 2 & 2 & 2 \\
\hline t Stat & 1.696156003 & 1.045508035 & -0.41978241 & 2.578180131 \\
\hline $\mathrm{P}(\mathrm{T}<=\mathrm{t})$ one-tail & 0.11597291 & 0.202764069 & 0.357720243 & 0.061620526 \\
\hline t Critical one-tail & 2.91998558 & 2.91998558 & 2.91998558 & 2.91998558 \\
\hline $\mathrm{P}(\mathrm{T}<=\mathrm{t})$ two-tail & 0.231945821 & 0.405528138 & 0.715440486 & 0.123241053 \\
\hline t Critical two-tail & 4.30265273 & 4.30265273 & 4.30265273 & 4.30265273 \\
\hline
\end{tabular}

\subsection{Experiment on the real image of the coronary artery}

The experiment on the cross-sectional images of the coronary artery have been carried out using 40 slices of IVUS. The maximum and minimum diameter of the lumen area were computed using the proposed algorithm. Samples of the images with the corresponding results are shown in Figure 6.
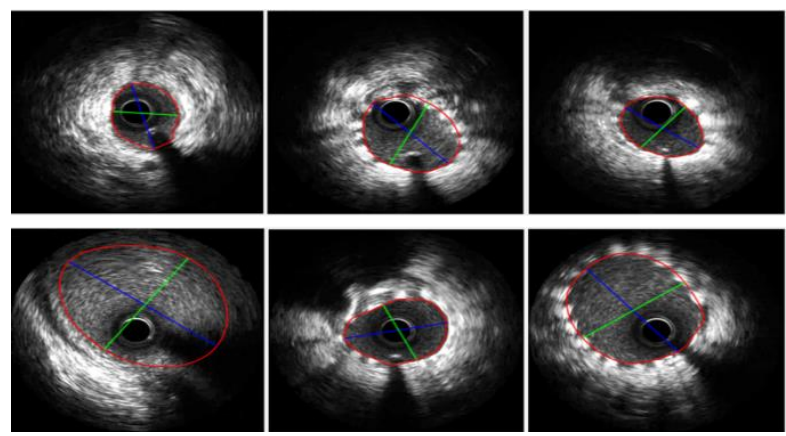

Figure 6. IVUS images with area (red), minimum (green) and maximum (blue) diameter computed using proposed algorithm 
The result have been compared with two different existing application which are "farthest_points.m", algorithm written by Matlab team in image processing tool and "distance calculator", application to calculate the distance between two points of coordinate geometry $(P, Q)$ using $(7)$.

$$
\operatorname{distance}(P, Q)=\sqrt{\left(x_{2}-x_{1}\right)^{2}+\left(y_{2}-y_{1}\right)^{2}}
$$

Table 4 compares the percentage difference between the experiment and the available methods to calculate area, minimum and maximum diameter. As can be seen from the table, the proposed method is compared to the farthest_points.m in term of area and maximum diameter only. The minimum diameter calculation not supported by the available method. For the second available method, only the distance between two points are available to be compared with the proposed method.

Table 4 . The percentage of difference between proposed method and existing methods

\begin{tabular}{ccc}
\hline & Experiment VS Method 1 & Experiment VS Method 2 \\
\hline Area & $0.00 \%$ & NIL \\
Min Diameter & NIL & $0.0005 \%$ \\
Max Diameter & $0.0015 \%$ & $0.0009 \%$ \\
\hline
\end{tabular}

The result revealed that the area calculation is accurate since there is no difference between the proposed method and the available method. For minimum diameter, the results only can be compared with the second available method and the percentage of difference is $0.0005 \%$. Comparison between the proposed method with the available methods on the maximum diameter, the value of the percentage difference is $0.0015 \%$ and $0.0009 \%$. All the comparison shows the small different between the proposed method and the existing methods indicate that the proposed method can be used as another alternative to measure unsymmetrical circle properties especially the area and diameter.

\subsection{Discussion}

In measuring the coronary artery, commercial software in the industry had provided us with the measurement. However, to our knowledge, no studies are available mentioned and described specifically the technique to measure the IVUS based on the image processing perspective as the purpose to help the diagnosis process. Most of the studies in IVUS segmentation measured the area of lumen only to compare the result with the ground truth experiment or with other findings $[23,24]$. In this study, we proposed the technique to measure CSA lumen, minimum and maximum diameter based on the previous border detection result. The disadvantage of this approach is the accuracy of the measurement was depended on the previously segmentation process. Nevertheless, in this study, the validation of the segmentation process was done thoroughly.

To ensure the computation process is correct, the technique has been tested on the object that we already knew the measurement. From the findings, it is approved that the proposed method is accurate. It is better if the findings of CSA, minimum and maximum diameter of the coronary artery to be compared with the available record however there is no available database that we can use for that purpose. Another source of uncertainty is in the method used to determine the calibration factor [25]. Because accuracy is very important in diagnosing medical images, we avoided making calibration factor using the different physical objects. In this study, we used IVUS's catheter shape as the reference image because the diameter is similar and accurate to the real catheter. The process has been suggested by the cardiologist. We managed to get an accurate calibration factor. After the measurement process was done, it is necessary to visualize the reading in graphical format. From the graphical presentation, the specialist could confirm their imaginary registration and the diagnosing analysis.

\section{CONCLUSION}

This paper highlights the importance of measuring the coronary artery cross-sectional image using image processing technique. Through the proposed computation of CSA, the minimum and maximum diameter of the lumen, the interpretation of the abnormalities that occurred on the artery could be confirmed by the cardiologist. As a result, treatment of errors such as not suitable stenting size used could be avoided. Besides that, the CSA, maximum and minimum diameter measurement could be used to measure another condition such as the velocity of the blood fluid and the thickness of the artery. The proposed algorithm could be used not only for the medical image but for other images in determining the area and the diameter of the object. 


\section{REFERENCES}

[1] A. M. Khairuddin, K. U. A. K. N. F, and P. E. H. Kan, "A general framework for improving electrocardiography monitoring system with machine learning," Bulletin of Electrical Engineering and Informatics, vol. 8, no. 1, pp. 261-268, 2019.

[2] Department of Statistics Malaysia, "Press Release Statistics On Causes Of Death, Malaysia, 2017 Deaths," 2017.

[3] D. Mozaffarian et al., "Heart disease and stroke statistics-2015 update: A report from the American Heart Association," Circulation, vol. 131, pp. 434-441, 2015.

[4] E. J. Benjamin et al., Heart Disease and Stroke Statistics'2018 Update: A Report from the American Heart Association, Circulation, vol. 135, pp. e67-e492, 2018.

[5] L. A. Pathak, S. Shirodkar, R. Ruparelia, and J. Rajebahadur, "Coronary artery disease in women," Indian Heart Journal, vol. 69, no. 4, pp. 532-538, 2017.

[6] Y. Kilic et al., "The Evolution of Data Fusion Methodologies Developed to Reconstruct Coronary Artery Geometry From Intravascular Imaging and Coronary Angiography Data: A Comprehensive Review," Frontiers in Cardiovasc Medicine, vol. 7, no. 33, pp. 1-13, 2020.

[7] T. Wan, H. Feng, C. Tong, D. Li, and Z. Qin, "Automated identification and grading of coronary artery stenoses with X-ray angiography," Computer Methods and Programs in Biomedicine, vol. 167, pp. 13-22, 2018.

[8] A. Vasquez, N. Mistry, and J. Singh, "Impact of Intravascular Ultrasound in Clinical Practice," International of Intravascular Ultrasound in Clinical Practice, vol. 9, no. 3, pp. 156-163, 2014.

[9] T. Nishi et al., "Comparison of 3-dimensional and 2-dimensional quantitative coronary angiography and intravascular ultrasound for functional assessment of coronary lesions," Journal of Cardiology, vol. 69, no. 1, pp. 280-286, 2017.

[10] C. V. Bourantas et al., "A new methodology for accurate 3-dimensional coronary artery reconstruction using routine intravascular ultrasound and angiographic data: Implications for widespread assessment of endothelial shear stress in humans," EuroIntervention, vol. 9, no. 5, pp. 582-593, 2013.

[11] L. Athanasiou et al., "Three-dimensional reconstruction of coronary arteries and plaque morphology using CT angiography - comparison and registration with IVUS," BMC Medical Imaging, vol. 16, no. 9, pp. 1-13, 2016.

[12] C. Doulaverakis et al., "IVUSAngio Tool: A publicly available software for fast and accurate 3D reconstruction of coronary arteries," Computers in Biology and Medicine, vol. 43, no. 11, pp. 1793-1803, 2013.

[13] E. I. Rentoukas and G. A. Lazaros, "Coronary intravascular ultrasound," Arch. Hell. Med., vol. 16, no. 1, pp. 29-38, 1999, doi: 10.1161/CIRCULATIONAHA.113.003534.

[14] A. Mantziari, A. Ziakas, G. Stavropoulos, and I. H. Styliadis, "Clinical applications of intravascular ultrasound (IVUS): Experience from an academic high volume centre of northern greece," Hippokratia, vol. 15, no. 1, pp. 60-63, 2011.

[15] S. B. Kutty, R. W. O. K. Rahmat, and S. S. Kassim, "Improved Segmentation for Intravascular Ultrasound ( IVUS ) Modality," International Journal of Engineering \& Technology, vol. 7, pp. 479-486, 2018.

[16] S. E. Nissen et al., "Intravascular ultrasound assessment of lumen size and wall morphology in normal Intravascular Ultrasound Assessment of Lumen Size and Wall Morphology in Normal Subjects and Patients With Coronary Artery Disease," Circulation, vol. 84, pp. 1087-1099, 1991.

[17] C. J. Marrocco et al., "Intravascular Ultrasound,” Seminars in Vascular Surgery, vol. 25, no. 3, pp. 144-152, 2012.

[18] M. A. G. Merkx, J. O. Bescõs, L. Geerts, E. M. H. Bosboom, F. N. Van De Vosse, and M. Breeuwer, "Accuracy and precision of vessel area assessment: Manual versus automatic lumen delineation based on full-width at halfmaximum," Journal of Magnetic Resonance Imaging, vol. 36, no. 5, pp. 1186-1193, 2012.

[19] S. A. Dhole and R. P. Shaikh, "Review of Leaf Unhealthy Region Detection Using Image Processing Techniques," Bulletin of Electrical Engineering and Informatics, vol. 5, no. 4, pp. 451-453, 2016.

[20] K. Mujika, J. Juanes, and A. Miguel, "Advantages and Disadvantages in Image Processing with Free Software in Radiology," Journal of Medical Systems volume, vol. 42, no. 36, 2018.

[21] J. Dijkstra, G. Koning, and J. H. C. Reiber, "Quantitative measurements in IVUS images," The International Journal of Cardiac Imaging, vol. 15, no. 6, pp. 513-522, 1999.

[22] V. C. Chijindu, C. C. Udeze, M. A. Ahaneku, and E. C. Anoliefo, "Detection of Prostate Cancer Using Radial / Axial Scanning of 2D Trans-rectal Ultrasound Images," Bulletin of Electrical Engineering and Informatics, vol. 7, no. 2, pp. 222-229, 2018.

[23] S. Balocco et al., "Standardized evaluation methodology and reference database for evaluating IVUS image segmentation," Computerized Medical Imaging and Graphics, vol. 38, no. 2, pp. 70-90, 2014.

[24] F. S. Zakeri, S. K. Setarehdan, and S. Norouzi, "Automatic media-adventitia IVUS image segmentation based on sparse representation framework and dynamic directional active contour model," Computers in Biology and Medicine, vol. 89, pp. 561-572, 2017.

[25] N. B. Byju and R. Rajesh Kumar, "Automated calibration of microscope based on image processing methods," Proceedings of the Fourth International Conference on Signal and Image Processing, pp. 91-101, 2012. 